\title{
CORRESPONDENCE
}

\section{Prognosis in pulmonary arterial hypertension}

\section{To the Editors:}

We read with interest the article by HUMBERT et al. [1] on the difference in prognosis in prevalent versus incident cohorts of patients with pulmonary arterial hypertension (PAH). The study highlights a survival bias in the prevalent cohort due to under-representation of more severely ill patients that might have died before inclusion in the registry. HUMBERT et al. [1] subsequently found a 1-yr and 3-yr survival rate of $89 \%$ and $69 \%$ for the prevalent cohort and $89 \%$ and $55 \%$ for the incident cohort of patients with idiopathic, familiar or anorexigenassociated $\mathrm{PAH}$, respectively.

Although we acknowledge that prevalent cohorts have a selection bias, it is surprising that this does not refer to a difference in mortality at 1-yr but only later during follow-up. If selection of the sickest patients would account for better survival one would expect the same phenomenon in the incident cohort. This would lead to a high early mortality rate, which then should stabilise over time as the sickest patients will have deceased. However, the prevalent patients have the disadvantage of potentially more advanced disease and, because of that, delayed start of targeted therapy.

In contrast to the results reported by HUMBERT et al. [1] we found a better survival for the incident patients when compared with the prevalent ones in a cohort of 250 patients with idiopathic, familiar or anorexigen-associated PAH. These patients were enrolled at seven German pulmonary hypertension centres between 1996 and 2008. All patients enrolled at the time of diagnosis were defined as incidental, whereas all remaining patients were defined as prevalent case. All underwent baseline invasive haemodynamic studies and the results as well as the baseline clinical data are shown in table 1.

Patients were subsequently started on targeted PAH therapy. Incident patients were started on inhaled iloprost $(n=11)$, oral beraprost $(n=8)$, bosentan $(n=21)$ or sildenafil $(n=6) .30$ patients were switched to a different PAH therapy after $301 \pm 67$ days and 22 patients were started on combined PAH therapy after $632 \pm 110$ days. Prevalent patients were started on inhaled iloprost $(n=66)$, intravenous iloprost $(n=10)$, oral beraprost $(n=24)$, bosentan $(n=56)$ or sildenafil $(n=2)$. 78 patients were subsequently switched to a different PAH therapy ( $422 \pm 46$ days) and 46 patients treated with combined PAH therapy $(807 \pm 91$ days).

During a mean follow-up of $1408 \pm 65$ days 20 of the incident patients and 71 of the prevalent patients died. 25 patients underwent lung transplantation (one in the incident group). Overall survival was significantly better in the incident group at year one, two and three (overall survival (95\% confidence interval) of $97.1 \%$ (93.1-100\%), 86.6\% (78.5-94.3\%) and $83.5 \%$ $(74.6-92.3 \%)$ in the incident group versus $83.5 \%$ (80.0-89.0\%), $74.2 \%(67.6-80.8 \%)$ and $67.8 \%(60.5-75.1 \%)$ in the prevalent group, all $\mathrm{p}<0.05$; event-free (death and transplant) survival $95.7 \%$ $(90.9-100 \%), 85.3 \%(76.9-93.7 \%)$ and $82.2 \%(73.1-91.3 \%)$ versus $79.9 \%(73.7-85.6 \%), 65.3 \%$ (58.2-72.4\%) and 57.4\% (50.0-64.8\%), all $\mathrm{p}<0.01)$. At 5 yrs, there was still a strong trend towards better overall survival (70.1 versus $57.9, \mathrm{p}=0.056)$ and a significantly better event-free survival (69.1 versus 45.0, $\mathrm{p}<0.001$ ) in the incident group.

Our data show an inverse prognostic relationship between incident and prevalent patients with idiopathic, familiar or

\section{TABLE 1 Baseline parameters of the study population}

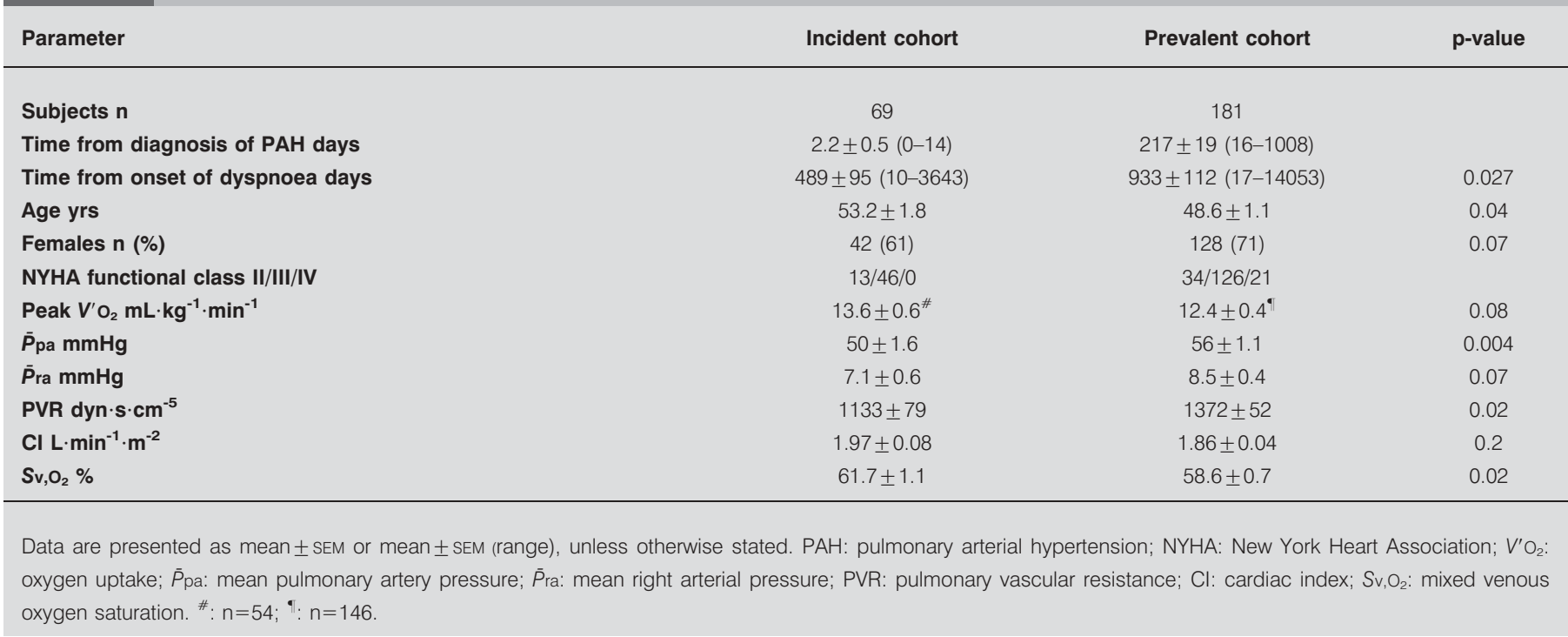


anorexigen-associated PAH. We would explain this observation by the substantial delay in the start of targeted $\mathrm{PAH}$ therapy in the prevalent cohort. This is confirmed by the prevalent patients showing more advanced disease as measured by invasive haemodynamics at the time of inclusion, as well as a trend towards reduced peak oxygen uptake. This observation is in agreement with data suggesting that patients suffer irreversible progression of disease if targeted therapy is delayed [2, 3]. Our data on the prognosis of incident patients also confirms previous survival data reported by HOEPER et al. [4] from a single centre cohort treated with contemporary targeted PAH therapy.

Even with cautious interpretation of our data, they strongly contrast with the generalised conclusions drawn by HUMBERT et al. [1] from their data. Although self-selection of sicker patients during the pre-inclusion phase in prevalent patients should result in a relative survival advantage of the remaining patients compared with incident patients [5], our data suggest that this can be offset by the beneficial effect of early institution of targeted therapy. Although comparing our data with those from HUMBERT et al. [1] is carrying some limitations, it illustrates the problem of generalising prognostic data even when obtained from a large multicentre registry.

\section{R. Wensel*, S. Gläser", C.F. Opitz ${ }^{*}$ and R. Ewert ${ }^{\#}$}

*International Centre for Circulatory Health, National Heart and Lung Institute, Imperial College London, London, UK. "Dept of Internal Medicine B, Ernst-Moritz-Arndt University Greifswald, Greifswald, and ${ }^{\top}$ DRK Kliniken Berlin Köpenick, Berlin, Germany.

Correspondence: R. Wensel, International Centre for Circulatory Health, National Heart and Lung Institute, Imperial College London, 59 North Wharf Road, London W2 1LA, UK. E-mail: rolandwensel@gmail.com

Statement of Interest: None declared.

\section{REFERENCES}

1 Humbert M, Sitbon O, Yaïci A, et al. Survival in incident and prevalent cohorts of patients with pulmonary arterial hypertension. Eur Respir J 2010; 36: 549-555.

2 Simonneau G, Rubin LJ, Galie N, et al. Addition of sildenafil to longterm intravenous epoprostenol therapy in patients with pulmonary arterial hypertension: a randomized trial. Ann Intern Med 2008; 149: 521-530.

3 Galiè N, Ghofrani HA, Torbicki A, et al. Sildenafil citrate therapy for pulmonary arterial hypertension. N Engl J Med 2005; 353: 2148-2157.

4 Hoeper MM, Markevych I, Spiekerkoetter E, et al. Goal-oriented treatment and combination therapy for pulmonary arterial hypertension. Eur Respir J 2005; 26: 858-863.

5 McLaughlin VV, Suissa S. Prognosis of pulmonary arterial hypertension: the power of clinical registries of rare diseases. Circulation 2010; 122: 106-108.

DOI: $10.1183 / 09031936.00171010$

\section{From the authors:}

We thank R. Wensel and colleagues for their comments on our recent article highlighting survivor bias in prevalent cohorts of pulmonary arterial hypertension (PAH) patients [1]. In this rebuttal, we provide information indicating that their analysis of our data and their own retrospective cohort probably suffer from misinterpretation and biases.

R. Wensel and colleagues report that a retrospective analysis from seven German pulmonary hypertension centres during 1996-2008 showed better survival in incident patients with idiopathic, familial or anorexigen-associated PAH patients compared to prevalent cases. This statement probably arises due to a misunderstanding of the definition of incident and prevalent cases. As stated in our recently published manuscripts [1-3], incident cases were defined as patients diagnosed with PAH using right-heart catheterisation during the recruitment phase of our registry. By contrast, prevalent cases were defined as patients in whom the diagnosis was made prior to the start of the registry. By definition, the date of diagnosis corresponded to the date of confirmatory right-heart catheterisation. We understand that R. Wensel and colleagues employed a different approach, defining so-called prevalent patients as subjects with a prior diagnosis of $\mathrm{PAH}$ in whom initiation of PAH-targeted therapy was delayed. This definition of prevalent patients erroneously leads the authors to state that "prevalent patients have the disadvantage of potentially more advanced disease and, because of that, delayed start of targeted therapy". Indeed, the distinction between incident and prevalent cases in our series by no means distinguishes a group of patients in whom initiation of treatment was delayed, as this would clearly be an unacceptable approach [4]. As stated in our articles, use of targeted therapies, including prostacyclin derivatives, endothelin receptor antagonists and phosphodiesterase 5 inhibitors was at the discretion of treating clinicians at each centre, according to current guidelines and availability of treatments [1, 3]. Conventional therapy alone was used in a similar proportion of incident and prevalent cases on PAH diagnosis (corresponding mostly to acute vasodilator responders who benefited from first-line calcium channel blocker therapy, patients in New York Heart Association functional class (NYHAFC) II or patients who died prematurely before any specific therapy could be proposed). In contrast, $70 \%$ of patients were treated with initial targeted therapy with a prostacyclin derivative (including intravenous epoprostenol), endothelin receptor antagonist or phosphodiesterase 5 inhibitors [2, 3].

In the letter of R. Wensel and colleagues, the authors refer to a retrospective analysis of 250 patients in seven German PAH centres over a 12-yr period. This relatively low number might reflect selection bias, as it is unlikely that expert centres treat, on average, only three incident and prevalent patients with idiopathic, familial or anorexigen-associated PAH annually. Recent European guidelines have attempted to define what would be the optimal characteristics of a pulmonary hypertension referral centre, and the proposed numbers were much higher than figures presented by R. Wensel and colleagues [4]. In the 2009 European Society of Cardiology/European Respiratory Society guidelines, it was stated that referral centres should follow $\geqslant 50$ patients with $\mathrm{PAH}$ or chronic thromboembolic pulmonary hypertension and should receive at least two new referrals per month [4]. In agreement with this recommendation, the French PAH Network (17 centres) recruited 674 $\mathrm{PAH}$ patients (356 idiopathic, familial and anorexigen-associated PAH) over a 1-yr period (October 2002-October 2003). 